\title{
Phthalocyanine Organic Thin-Film Transistors for the Detection of dimethyl methyl phosphonate (DMMP)
}

\author{
Huiling Tai*, Guangzhong Xie, Xian Li, Bo Zhang, Wenchao Dan \\ State Key Laboratory of Electronic Thin Films and Integrated Devices, School of Optoelectronic \\ Information, University of Electronic Science and Technology of China (UESTC), Chengdu Jianshe \\ North Road, section2 No.4, 610054, P. R. China
}

\begin{abstract}
:
The electrical properties of Metal-Phthalocyanines (M-Pc) are affected upon exposure to gases containing not only some oxidizing/deoxidizing molecules but also nerve agent simulants. This phenomenon finds application in the use of Organic Thin-Film Transistors (OTFTs) based on copper phthalocyanine (CuPc) and cobalt phthalocyanine (CoPc) as gas sensors. In this work, tests of dimethyl methyl phosphonate (DMMP) detecting were carried out, and the sensing mechanism of OTFT DMMP sensors was further investigated by the transfer curve of OTFTs and the UV-Vis absorption spectra of sensitive films before and during DMMP vapor exposure. The results showed that M-Pc-based OTFTs can represent a new class of DMMP sensors.
\end{abstract}

Key words: OTFTs, CuPc, CoPc, gas sensors and DMMP

\section{Introduction}

The use of chemical weapons against civilians by terrorist groups or fanatic individuals is not just horror fiction anymore, but an absolute real threat [1]. The fast and accurate detection of chemical warfare agents (CWAs) is essential to protect human beings, thus many researchers have already attempted to detect toxic gases using various kinds of sensors. Among them, organic thin-film transistors (OTFTs) offer a great deal of promise for application in chemical and biological sensing [2]. For the detection of CWAs, there is a demand for small, portable, and inexpensive sensors. OTFTs have many advantages over other types of sensors that may allow them to meet these needs, such as their ease of deposition, ability to operate at room temperature and the multi-dimensional patter [34]. Therefore, there has been an increasing progress in OTFT performances during the last decade.

Metal-Phthalocyanine (M-Pc) is known to be very sensitive and also selective to different gases and solvents as an account of the central metal atoms of the molecule, which can be easily varied. Phthalocyanine especially, due to their highly thermal stability, posses a wide range of chemical and physical properties, that make them interesting as an active layer in OTFTs [3]. Over the past decade, the use of M$\mathrm{Pc}$ as a sensitive component has been investigated for many kinds of gases [56].However, to the best of our knowledge, there were few reports for the DMMP sensor using the $\mathrm{M}-\mathrm{Pc}$ as the sensitive materials. R. D. Yang and J. Park had developed a pulsed gating method to enhance the baseline stability of OTFT chemical sensors based on the M-Pc, and the analytes employed were methanol, DIMP and DMMP [7].

This paper describes the preparation of a gas sensor based on OTFTs obtained from vacuum-deposition copper phthalocyanine (CuPc) and cobalt phthalocyanine (CoPc) on a thermally grown $\mathrm{SiO}_{2} / \mathrm{Si}$ wafer. The saturation current of OTFTs was used as a good parameter for monitoring the dimethyl methyl phosphonate (DMMP) gas, the simulant of nerve agents, and the gas-sensing mechanism of OTFTs was further investigated.

\section{Experimental}

The OTFT was fabricated using standard semiconductor processing techniques. The channel length and width is 10 and $4000 \mu \mathrm{m}$, respectively. The insulating layers $\mathrm{SiO}_{2}$ is 195 $\mathrm{nm}$ in thickness. Titanium (Ti) $(20 \mathrm{~nm})$ and gold (Au) $(50 \mathrm{~nm})$ bilayer was sputtered on top of the gate dielectric layer as the source and drain electrodes. The CuPc and $\mathrm{CoPc}$ were purchased (purity $>99.5 \%$ ) and used without any purification, which was deposited by vacuum deposition at a average deposition rate of 0.3 
Ås. The substrate temperature was maintained at $260^{\circ} \mathrm{C}$, and the thickness of the active layer is about $90 \mathrm{~nm}$.

The layout of an OTFT sensing experiment was shown in Fig.1, illustrating the delivery of DMMP molecules from the sample reservoir to the surface of the OTFT gas sensor. $100 \mathrm{ppm}$ standard DMMP gases were obtained, and the gas concentration is controlled by the mass flow controls. The source-drain voltage and the gate voltage were both swept between 0 and $-60 \mathrm{~V}$. The saturation source-drain currents were recorded as the output signals of sensors using a Keithley 4200-SCS source measurement unit at room temperature.

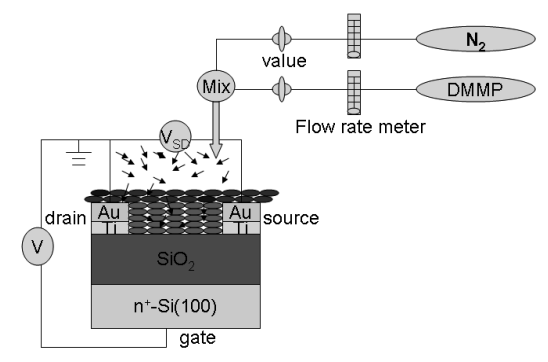

Fig. 1. Schematic system of DMMP delivery with OTFT under experiment

The UV-vis absorption spectra of phthalocyanine thin films were recorded on a Shimadzu UV1700 spectrometer using an uncoated glass as the reference. The surface morphology was assessed with a Hitachi S4800 scanning electronic microscope (SEM).

\section{Microstructure Characterization}

It is well-known that the surface morphologies and the grain boundaries of organic thin films can influence the sensing property of OTFT sensors [8]. Fig. 2 shows the SEM images of CuPc (a) and CoPc (b) thin films, which both indicates the plate-like structures, with the topmost layer of plates standing perpendicular to the surface. For the gas sensor application, surface structures like this film are desired because of the large surface area and more adsorption sites compared to films with large bulk grains. (a) $\mathrm{CuPc}$

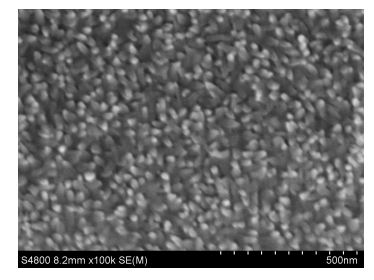

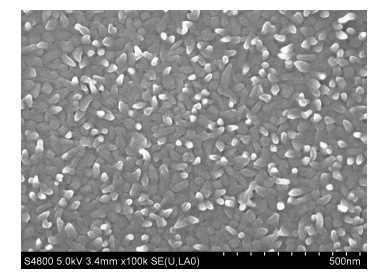

(b) $\mathrm{CoPC}$
Fig. 2. The SEM images of M-Pc thin films

\section{Current voltage characteristics of OTFT}

The $I_{d}-V_{d}$ characteristics of CuPc and CoPc OTFT sensors are shown in Fig.3, which exhibited the obvious linear and saturation regions. It was noticeable that the source-drain current of CuPc OTFT sensor was apparently larger than that of CoPc OTFT sensor. It was because that the nature of the sequestered metal ions has an influence on the physicochemical properties of the M-Pc. And the results indicated that the conductivity of the $\mathrm{CuPc}$ thin film was superior to that of CoPc film. For the sensor application, it is more desirable to have a higher source-drain current, so that the CoPc OTFT was not very suitable for the DMMP sensor compared with CuPc OTFT in this sense.
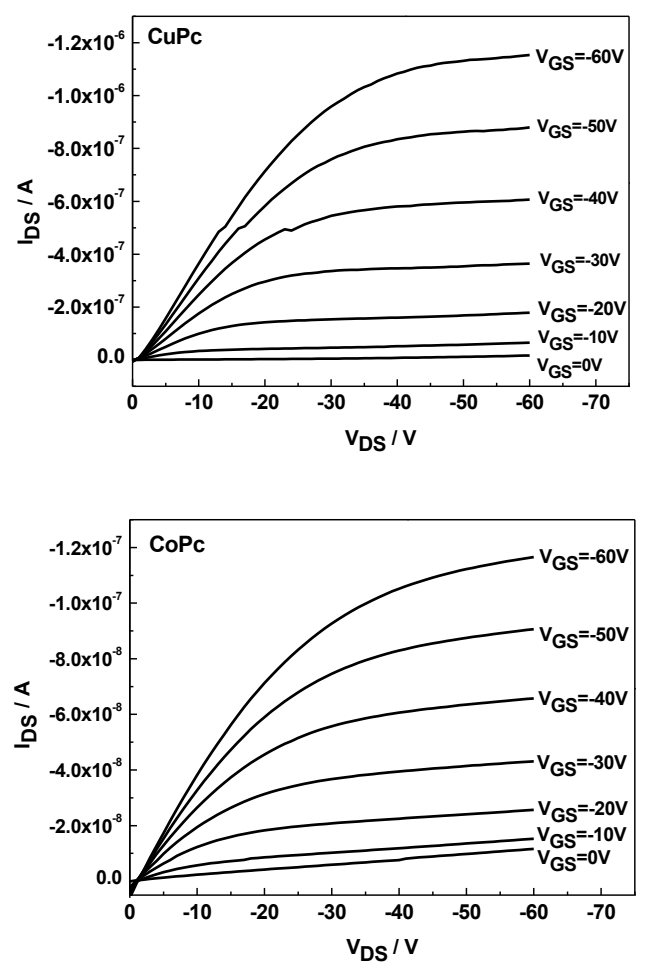

Fig. 3. $I_{d}-V_{d}$ characteristics of CuPc and $\mathrm{CoPc}$ OTFT sensors

\section{Performance of OTFT sensors}

Fig.4 showed the changes in drain-source current as a function of time when the CuPc and CoPc OTFT sensors were exposed to different exposure/evacuation cycles of DMMP gases in concentrations ranging from 40 to $80 \mathrm{ppm}$ at room temperature. The measurements of the time dependence of the drain-source current in the OTFT sensors were made at a gate voltage of $-40 \mathrm{~V}$ and a drain-source voltage of $-50 \mathrm{~V}$. As can be seen in Fig.4, the drain-source current decreased rapidly whereas it could not recover completely to its original baseline. The results also indicated that the conductivity and the 
response of the CuPc thin film were all superior to those of the CoPc film.
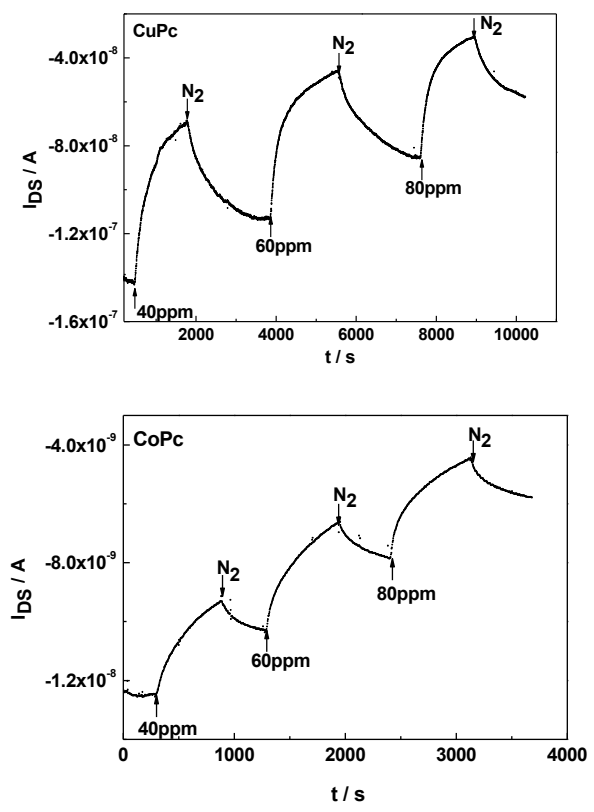

Fig. 4. Response transients of the prepared OTFT sensors to DMMP

\section{Sensing mechanism}

Fig. 5 plotted the square root of the saturation drain current of CuPc and CoPc OTFT sensors versus gate voltage before and during 50 ppm DMMP gas exposure.
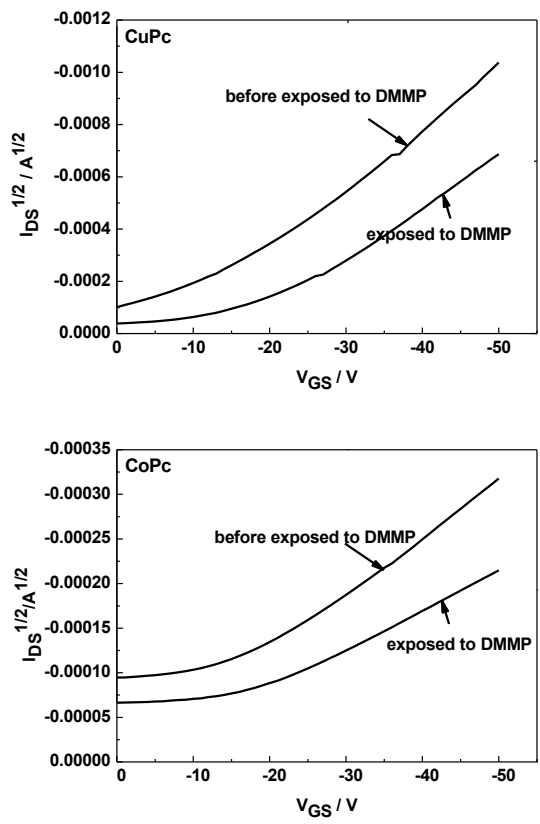

Fig. 5. Square root of the saturation drain current of CUPc and CoPc OTFT sensors versus gate voltage before and during DMMP gas exposure

It was remarkable that the source-drain current was not zero at zero gate bias, which was because that thermally evaporated organic thin films are unintentionally $\mathrm{p}$ or $\mathrm{n}$ doped and only highly pure materials originate TFTs that are off already at zero gate bias.

The threshold-voltage $\left(V_{T}\right)$ was extracted from the measured drain current versus gate voltage transfer characteristics by the linear extrapolation, and the changes of the thresholdvoltage $\left(\Delta \mathrm{V}_{\mathrm{T}}\right)$ of CuPc and CoPc OTFT sensors were shown in Tab. 1 and Tab. 2, respectively. It can be seen that the $V_{T}$ value shifted further toward the negative direction in comparison to the $V_{T}$ of standard OTFT sensors without exposure to DMMP gas. Since the shift in $V_{T}$ can be estimated according to Poisson's equation [15]:

$$
\Delta V_{T}=\frac{-N \mu_{I}}{\varepsilon}
$$

Where $\mathrm{N}$ is the density of the adsorption site, $\mu_{I}$ is the number of dipole moments, and $\varepsilon$ is the permittivity of the organic layer. Therefore, the $\Delta \mathrm{V}_{\mathrm{T}}$ may be ascribed to the interactions between the dipolar molecules adsorbed on the active layer or adsorption sites between grain boundaries of the $\mathrm{Pc}$ thin films, and was related to the change of the work function at the interface between the organic semiconductor layer and the insulator, induced by the adsorption of the polar molecules. The variations in the field-effect mobility $\left(\Delta \mu_{\text {sat }}\right)$ of OTFT sensors with and without exposure to DMMP (50 ppm) are also summarized in Tab. 1 and Tab.2, which indicated that the $\mu_{\text {sat }}$ values decreased when OTFT sensors were exposed to DMMP compared with those not exposed to the gas.

Tab. 1: The values for CUPc OTFT parameters such as $V_{T}$ and $\mu_{\text {sat }}$ as functions of DMMP gases

\begin{tabular}{|c|c|c|}
\hline & $\begin{array}{c}\text { CuPc } \\
\text { (no DMMP) }\end{array}$ & $\begin{array}{c}\text { CuPc } \\
\text { (50ppm DMMP) }\end{array}$ \\
\hline $\mathrm{V}_{\mathrm{T}}(\mathrm{V})$ & -9 & -16 \\
\hline$\mu_{\mathrm{sat}}\left(\mathrm{cm}^{2} / \mathrm{V} \cdot \mathrm{s}\right)$ & $-1.16 \times 10^{-4}$ & $-0.44 \times 10^{-4}$ \\
\hline$\Delta \mathrm{V}_{\mathrm{T}}(\mathrm{V})$ & \multicolumn{2}{|c|}{-7} \\
\hline$\Delta \mu_{\mathrm{sat}}\left(\mathrm{cm}^{2} \mathrm{~V} \cdot \mathrm{s}\right)$ & \multicolumn{2}{|c|}{$0.72 \times 10^{-4}$} \\
\hline
\end{tabular}

Tab. 2: The values for CoPc OTFT parameters such as $V_{T}$ and $\mu_{\text {sat }}$ as functions of DMMP gases

\begin{tabular}{|c|c|c|}
\hline & $\begin{array}{c}\text { CoPc } \\
(\text { no DMMP })\end{array}$ & $\begin{array}{c}\text { CoPc } \\
(50 p p m ~ D M M P)\end{array}$ \\
\hline $\mathrm{V}_{\mathrm{T}}(\mathrm{V})$ & -2.0 & -3.5 \\
\hline$\mu_{\mathrm{sat}}\left(\mathrm{cm}^{2} / \mathrm{V} \cdot \mathrm{s}\right)$ & $-1.15 \times 10^{-5}$ & $-0.53 \times 10^{-5}$ \\
\hline$\Delta \mathrm{V}_{\mathrm{T}}(\mathrm{V})$ & \multicolumn{2}{|c|}{-1.5} \\
\hline$\Delta \mu_{\mathrm{sat}}\left(\mathrm{cm}^{2} \mathrm{~V} \cdot \mathrm{s}\right)$ & \multicolumn{2}{|c|}{$-0.62 \times 10^{-5}$} \\
\hline
\end{tabular}


It was believed that the change of saturation drain current can be attributed to the variation of both threshold voltage and field effect mobility [8], hence the changes of these two parameters are consistent with those of drain currents.

Fig. 6 displays the UV-vis absorption spectra of CoPc and CuPc thin films before and during DMMP gas exposure. The CuPc thin film shows the bands at about $615 \mathrm{~nm}$ and $690 \mathrm{~nm}$ and the CoPc thin film shows the bands at about $610 \mathrm{~nm}$ $680 \mathrm{~nm}$, which should be due to the $n-\pi^{*}$ transition. From the plots shown in Fig.6, it can be noted that there was no new absorption band appeared, and the exposure to DMMP only resulted in the change of the absorption values, indicating a physical absorption, probably a weak electrostatic force of the van der Waals type in the active film, and no chemical interaction or the structural change occurred. Therefore, the change in currents observed when OTFT sensors were exposed to DMMP should be a combination of effects on charge carrier mobility and threshold voltage based on the above measurement and analysis.
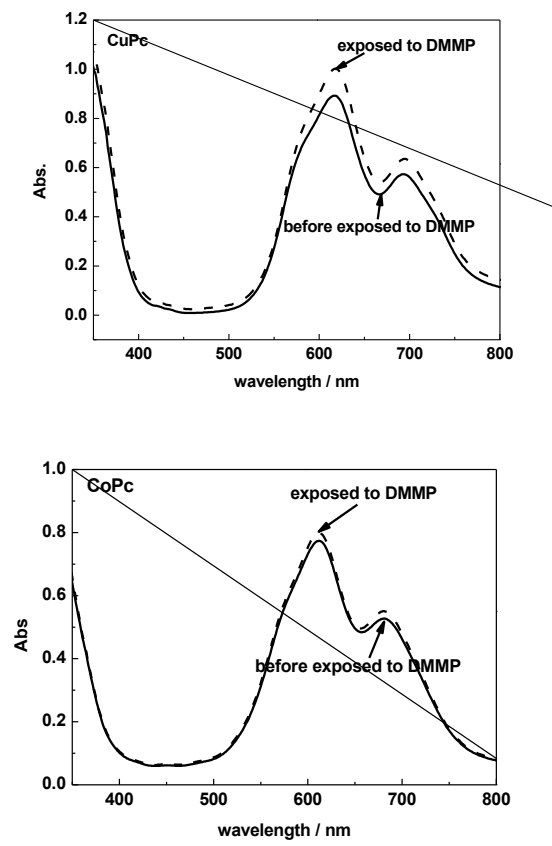

Fig. 6. UV-Vis absorption spectra of CuPc and CoPc thin films before and during DMMP gas exposure

\section{Conclusion}

The DMMP sensors had been prepared based on OTFTs made from vacuum deposited CuPc and $\mathrm{CoPc}$ thin films on thermally grown $\mathrm{SiO}_{2} / \mathrm{Si}$ wafers. The time dependence of the drainsource current had been observed when exposed to DMMP gases ranging from 40-80 ppm, which showed that the response characteristics of CuPc OTFT sensors were superior to those of CoPc OTFT sensors. Also, the response characteristics of the OTFT DMMP sensors had shown results in the thresholdvoltages being shifted and a decrease in the field-effect mobility, and no chemical interaction or the structural change occurred by the UV-vis absorption spectra testing. Therefore, the change in currents should be a combination of effects on charge carrier mobility and threshold voltage.

\section{Acknowledgements}

This work was supported by National Science Foundation of China via grants No.61101031 and No.61101029, and the Fundamental Research Funds for the Central Universities via grants No. ZYGX2009J052.

\section{References}

[1] A. A. Tomchenko, G. P. Harmer, B. T. Marquis, Detection of chemical warfare agents using nanostructured metal oxide sensors, Sensors and Actuators B 108, 41-55 (2005); doi: 10.1016/j.snb.2004.11.059

[2] J.T. Mabeck, G.G.Malliaras, Chemical and biological sensors based on organic thin film transistors, Analytical and Bioanalytical Chemistry,384,343-353 (2006); doi: 10.1007/s00216-005-3390-2

[3] R. B. Chaabane, Adne`ne Ltaie, L. Kaabi, H. Ben Ouada, N. Jaffrezic-Renault, J. Davenas, Influence of ambient atmosphere on the electrical properties of organic thin film transistors, Materials Science and Engineering C, 26, 514-518 (2006); doi: 10.1016/j.msec.2005.10.018

[4] L. Torsi, A. Dodabalapur, L. Sabbatini, P.G. Zambonin, Multi-parameter gas sensors based on organic thin-film-transistors, Sensors and Actuators $B, \quad 67, \quad 312-316 \quad$ (2000); doi: 10.1016/S0925-4005(00)00541-4

[5] M. C. Tanese, D. Fine, A. Dodabalapur, L. Torsi, Interface and gate bias dependence responses of sensing organic thin-film transistors, Biosensors and Bioelectronics, 21, 782-788 (2005);doi:10.1016/j.bios.2005.01.016

[6] R.B. Chaabane, Effect of measuring environment on the electrical characteristics of NiPc based thin film transistors: The effects of ozone, Materials Science and Engineering C, 26,551-554 (2006);doi:10.1016/j.msec.2005.10.012

[7] R. D. Yang, J. Park, Ultralow drift in organic thinfilm transistor chemical sensors by pulsed gating, Journal of Applied Physics, 102, 034515 (2007), doi: 10.1063/1.2767633

[8] J. Huang, J. Miragliotta, A. Becknell, H. E. Karz, Hydroxy-Terminated Organic SemicondutorBased Field-Effect Transistors for Phosphonate Vapor Detection, Journal of the American Chemical Society, 129, 9366-9376 (2007),doi: 10.1021/ja068964z 